\title{
MMX geodesy investigations: science requirements and observation strategy
}

\author{
Koji Matsumoto ${ }^{1,2^{*}}$ (0), Naru Hirata ${ }^{3}$, Hitoshi Ikeda ${ }^{4}$, Toru Kouyama ${ }^{5}$, Hiroki Senshu ${ }^{6}$, Keiko Yamamoto ${ }^{7}$,
} Hirotomo Noda ${ }^{1,2}$, Hideaki Miyamoto $^{8}$, Akito Araya ${ }^{9} \mathbb{D}$, Hiroshi Araki ${ }^{7}$, Shunichi Kamata ${ }^{10}$, Nicola Baresi ${ }^{4,11}$ and Noriyuki Namiki ${ }^{2,7}$

\begin{abstract}
In order to investigate the origin of Phobos and Deimos, the Japanese Martian Moons eXploration (MMX) mission is scheduled for launch in 2024. MMX will make comprehensive remote-sensing measurements of both moons and return regolith samples from Phobos to Earth. Geodetic measurements of gravity, shape, and rotation parameter of a body provides constraints on its internal structure reflecting its origin and evolution. Moments of inertia are important parameters to constrain the internal mass distribution, but they have not been well determined for the Martian moons yet. We discuss the mission requirements related to the moments of inertia to detect a potential heterogeneity of the mass distribution inside Phobos. We introduce mission instruments and operational strategies to meet the mission requirements. We present a preliminary imaging strategy from a quasi-satellite orbit for a base shape model that is expected to be created at the early stage of the mission. Geodetic products including ephemeris, gravity field, rotation parameter of Phobos, and spacecraft orbit are of importance not only for the geodetic study, but also for interpreting data from various mission instruments and selecting possible landing sites.
\end{abstract}

Keywords: MMX, Gravity, Shape, Rotation, Internal structure

\section{Graphical Abstract}

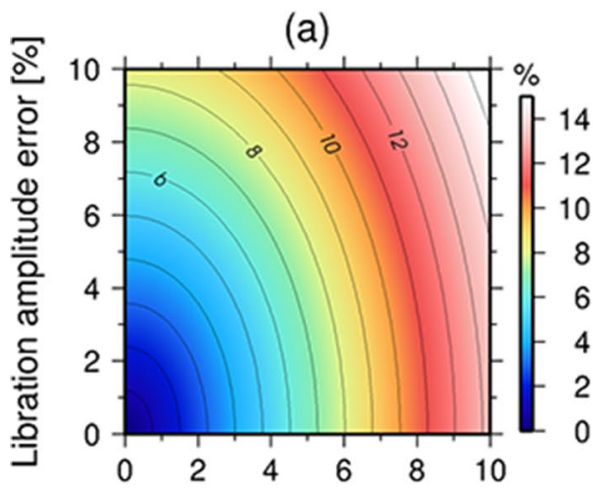

Gravity coefficient error [\%]

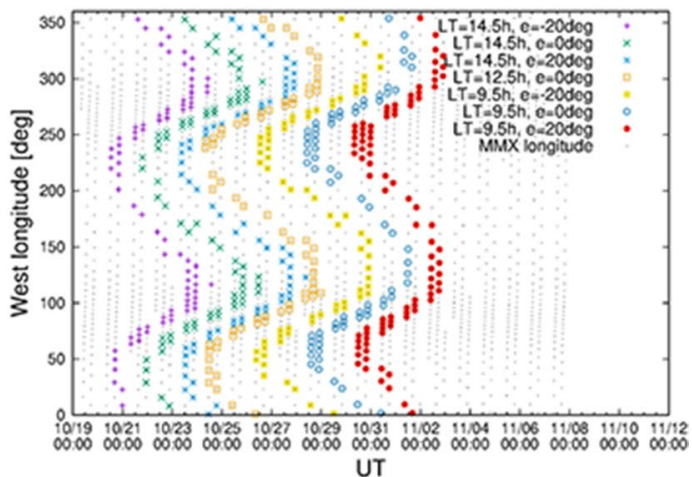

UT

\footnotetext{
*Correspondence: koji.matsumoto@nao.ac.jp

${ }^{1}$ RISE Project, National Astronomical Observatory of Japan, Oshu, Iwate 023-0861, Japan

Full list of author information is available at the end of the article
}

\section{Introduction}

The origin of Phobos is still an open issue. It may either be a captured asteroid or have formed from a disk of impact ejecta produced by a giant impact (Craddock 
2011). The Martian Moons eXploration (MMX) led by Japan Aerospace Exploration Agency (JAXA) will provide a unique opportunity to answer this question from various aspects including remote-sensing observations and returned samples (Kawakatsu et al. 2019; Kuramoto et al. 2021). MMX is scheduled for launch in 2024 and will make progress in our understanding of planetary system formation as well as primordial material transport between the inner- and the outer-part of the early solar system. The internal structure can possibly provide clues on the formation regime and thus on the origin of Phobos. Although it is not straightforward to determine the origin from the internal structure alone, the internal structure provides hints to the origin and the sequence of evolution. Because of its small size and mass, Phobos could have a very puzzling interior structure (Le Maistre et al. 2019). It is expected that the low density $(\sim 1847 \mathrm{~kg} /$ $\mathrm{m}^{3}$, "Bulk density and moments of inertia" section) of Phobos is due to the presence of significant macro-porosity and/or water ice in its interior, in both cases the distribution depends on the formation scenario. The presence or absence of such deviations from a homogenous mass distribution will help us trace back the origin of the satellite.

Geodetic observations of shape, gravity and rotation parameter contribute to detecting the signatures of a heterogeneous interior and providing constraints on internal structure models. We can expect larger signatures from the geodetic observations for larger heterogeneous mass distribution. Some of the key parameters related to the internal structure are the moments of inertia (MOI) which constrain the radial distribution of mass within the interior. MOI are not directly measurable, but can be determined by combining measurements of rotation parameters with measurements of degree- 2 gravity coefficients. A rotation parameter that is particularly important for description of Phobos' rotation is the amplitude of short period forced libration in longitude (e.g., Burmeister et al. 2018). Currently, the MOI are not estimated with sufficient accuracy mainly due to large errors in the gravity coefficients (e.g., Pätzold et al. 2014; Jacobson and Lainey 2014; Yang et al. 2019).

In this paper, we review recent geodetic observations of Phobos, describe geodetic mission requirements related to the MOI using a two-layered Phobos model, and show preliminary observation strategies for the geodetic observations with special emphasis on shape modeling. This paper focuses on observations of Phobos. Observations of Deimos will be discussed possibly later after the design of a flyby trajectory has progressed.

\section{Present state of geodetic observation of Phobos Shape model}

A global shape/topography model can be used to calculate the MOI based on various internal structure models. It may also provide insight into surface processes and regolith evolution (e.g., Miyamoto et al. 2007; Ballouz et al. 2019) and together with gravity models constrain the orbital evolution (Hu et al. 2020). Furthermore, an accurate shape/topography models is also necessary for the photometric correction of images, the identification of potential landing sites having minimal surface hazard, the provision of landmarks for image-based spacecraft orbit determination (Konopliv et al. 2018), and the provision of a reference shape to which an altimeter-measured topographic profile is geometrically fitted to improve spacecraft position (Matsumoto et al. 2020), etc.

Because the shape of a body is essential as described above, much shape and topographic modeling effort on Phobos has been made since explorations of the Martian system started. Willner et al. (2010) introduced a shape model in the form of a digital terrain model (DTM) and a ground control point (GCP) network that was derived from images of the super resolution channel (SRC) of the high-resolution stereo camera (HRSC) on Mars Express (MEX). The GCPs of a planetary body are points of which coordinates are known with respect to the bodyfixed rotating frame. The mean accuracy of the GCPs are $21.24 \mathrm{~m}, 16.19 \mathrm{~m}$, and $16.70 \mathrm{~m}$ for XYZ axes of the Phobos body-fixed coordinate, respectively. Willner et al. (2014) then introduced a newer shape model with $100 \mathrm{~m} /$ pixel resolution and a set of spherical harmonic coefficients to degree and order 45 estimated from images of the SRC and the framing camera on the Viking Orbiter (VO) with the stereophotogrammetry (SPG) method. Although they did not mention the quantitative accuracy of the 2014 version of the shape model, they stated that the model shows a better representation of Phobos' surface features than their previous model of Willner et al. (2010).

Gaskell (2011) also released another shape model of Phobos through NASA Planetary Data System (PDS). This model was originally reconstructed from $224 \mathrm{VO}$ images and 8 Phobos 2 images in 2006 by the stereophotoclinometry (SPC) method (Gaskell et al. 2008). The model consists of over 3 million triangle plates with the average length of plates of $35.5 \mathrm{~m}$. The data producer gives the RMS residual error of landmarks forming the shape model of $25 \mathrm{~m}$.

Ernst et al. (submitted to Earth Planets and Space) created high-resolution SPC shape models of Phobos and 
Deimos with images taken by six spacecraft from five missions of Viking Orbiter, Phobos 2, Mars Global Surveyor, Mars Express, and Mars Reconnaissance Orbiter. These models are publicly available in the Small Body Mapping Tool (SBMT, Ernst et al. 2018) distributed at the SBMT website (https://sbmt.jhuapl.edu) and will be archived with the NASA Planetary Data System.

\section{Gravity field}

The MEX spacecraft orbits Mars in a highly elliptical and quasi-polar orbit, which provides opportunities to perform close flybys of Phobos every 150 days on average (Witasse et al. 2014). Andert et al. (2010) estimated GM (the product of the Newtonian constant of gravitation $G$ and the body's mass $M$ ) of Phobos from MEX tracking data during its close flyby in 2008 at $275 \mathrm{~km}$ distance. Their solution is $G M=(0.7127 \pm 0.0021) \times 10^{-3}$ $\mathrm{km}^{3} / \mathrm{s}^{2}$ with a quoted $1 \sigma$ accuracy of $0.3 \%$. Pätzold et al. (2014) attempted to estimate degree-2 gravity coefficients $C_{20}$ and $C_{22}$ along with $G M$ of Phobos from data during another closer flyby in 2010 at $77-\mathrm{km}$ distance. The updated $G M$ value is $(0.7072 \pm 0.0013) \times 10^{-3} \mathrm{~km}^{3} / \mathrm{s}^{2}$ $(3 \sigma)$ with five times better accuracy than that of the 2008 flyby. However, degree- 2 gravity coefficients could not be solved for at sufficient accuracy: $C_{20}=-0.1145 \pm 0.1456$ $(3 \sigma)$ and $C_{22}=0.0025 \pm 0.0056(3 \sigma)$. They explained that the high error of the coefficients is due to strong correlation of the three parameters of $G M, C_{20}$ and $C_{22}$. Yang et al. (2019) analyzed MEX tracking data of two combined flybys in 2010 and 2013 and estimated $G M, C_{20}$, and $C_{22}$. The closest approach distance in 2013 flyby was $59 \mathrm{~km}$. They used the result of Shi et al. (2012), who calculated gravity coefficients based on a shape model and constant density assumption, as a priori information. Their results are $G M=(7.0765 \pm 0.0075) \times 10^{5} \mathrm{~m}^{3} \mathrm{~s}^{-2}$ $(3 \sigma), C_{20}=-0.1378 \pm 0.0348(3 \sigma)$ and $C_{22}=0.0166 \pm$ $0.0153(3 \sigma)$. The corresponding $1 \sigma$ error is $0.04 \%, 8 \%$ and $31 \%$, respectively. The better accuracy for $C_{20}$ seems to be due to the decorrelation of the adjusted parameters owing to the two independent flybys at different geometries and due to the closer flyby distance in 2013 than that in 2010.

The orbit of Phobos is sensitive to its own aspherical gravity field, allowing for the estimation of Phobos' gravity coefficients from observation of its orbital dynamics. Jacobson and Lainey (2014) applied direct numerical integration method and fitted $C_{20}$ and $C_{22}$ of Phobos together with the Phobos and Deimos ephemeris and GMs using Earth-based astrometric observations since 1877 and spacecraft-based imaging and Doppler tracking data. They emphasize that they did not estimate the amplitude of forced libration of Phobos, but adopted the value derived by Willner et al. (2010). The estimated gravity field coefficients are $C_{20}=-0.1057 \pm 0.0067$ and $C_{22}=0.01487 \pm 0.0004$. The reported $1 \sigma$ accuracies are about $6 \%$ and $3 \%$ for $C_{20}$ and $C_{22}$, respectively, but they are estimated with a correlation of 0.96 indicating that the estimates are not independent. Jacobson (2010) pointed out that only one of the three parameters $C_{20}, C_{22}$, and libration amplitude can be independently estimated from the observed secular rate of periapsis longitude.

\section{Bulk density and moments of inertia}

The mass of Phobos calculated from the GM value of Yang et al. (2019) and $G=(6.67408 \pm 0.00047) \times 10^{-11} \mathrm{~m}^{3} \mathrm{~kg}^{-1} \mathrm{~s}^{-2} \quad($ Mohr et al. 2016) is $M=(1.0603 \pm 0.0004) \times 10^{16} \mathrm{~kg}$. The $1 \sigma$ uncertainty of the Phobos' mass is about $0.04 \%$ for which the uncertainty of $G M$ is more dominant than that of $G$. The bulk density of Phobos is $\rho=(1847 \pm 12) \mathrm{kg} / \mathrm{m}^{3}$ by applying the volume $V=(5742 \pm 35) \mathrm{km}^{3}$ calculated from the shape model of Willner et al. (2014). Currently, the error of the bulk density of about $0.6 \%$ is dominated by the error of the volume.

Moments of inertia are of interest in developing models of the satellite's interior structure. They are derived from the amplitude of forced libration in longitude and degree-2 gravity coefficients $\left(C_{20}, C_{22}\right)$ of Phobos. If the principal moments of inertia are denoted $A<B<C$, the libration amplitude is expressed as (e.g., Duxbury and Callahan 1989; Willner et al. 2010)

$$
\theta=\frac{2 e}{\left(1-\frac{1}{3 \gamma}\right)},
$$

where $e=0.01511$ (Jacobson 2010) is the orbital eccentricity of Phobos and $\gamma$ is the dynamical flattening

$$
\gamma=\frac{B-A}{C} \text {. }
$$

It is not possible to determine the absolute value of $A$, $B$, and $C$ from $\theta$ alone. On the other hand, degree- 2 gravity coefficients are related to the MOI, mass $M$, and mean radius $R_{0}$ as follows (e.g., Bills and Rubincam 1995):

$$
\begin{aligned}
& C_{20}=\frac{(B+A)-2 C}{2 M R_{0}^{2}}, \\
& C_{22}=\frac{(B-A)}{4 M R_{0}^{2}} .
\end{aligned}
$$

Then, by combining $\gamma$ as a function of $\theta$ and $\left(C_{20}, C_{22}\right)$, each of dimensionless MOI normalized by $M R_{0}^{2}$ can be derived as follows (e.g., Borderies and Yoder 1990): 


$$
\begin{aligned}
& \frac{A}{M R_{0}^{2}}=\frac{\gamma C_{20}+2(2-\gamma) C_{22}}{\gamma}, \\
& \frac{B}{M R_{0}^{2}}=\frac{\gamma C_{20}+2(2+\gamma) C_{22}}{\gamma}, \\
& \frac{C}{M R_{0}^{2}}=\frac{4 C_{22}}{\gamma},
\end{aligned}
$$

where $\gamma$ is given by Eq. (1). Because of the large uncertainties of degree- 2 gravity coefficients, moments of inertia have not been obtained yet.

\section{Amplitude of forced libration in longitude}

The rotation parameters of a planetary body describe the time-varying orientation of the body-fixed rotating reference frame relative to an inertial reference frame. One of the parameters that is of interest with regard to the internal mass distribution of Phobos is the amplitude of forced libration in longitude. Phobos is in synchronous spin-orbit resonance and facing the same side to Mars on average. Superimposed on the uniform mean rotation are oscillations called physical librations that are caused by time-varying gravitational torque that Mars exerts on the dynamical figure of Phobos. The longitudinal libration, an east-west oscillation of the body's surface about the spin axis, has been observed by spacecraft.

The libration amplitude has been estimated from GCP network analysis using multiple image data. Willner et al. (2010) analyzed image data obtained by the SRC of the HRSC on board MEX and VO framing camera to compute coordinates of 665 GCPs together with the forced libration amplitude of Phobos. They tested various plausible values of the libration amplitude and determined a best fitting value of $\theta=-1.2^{\circ} \pm 0.15^{\circ}$ that gives the smallest mean residual of the GCPs. Oberst et al. (2014) updated the results with 813 GCPs and derived a libration amplitude of $\theta=-1.09^{\circ} \pm 0.10^{\circ}$. Burmeister et al. (2018) developed a mathematical framework to directly solve for rotation parameters in the inertial reference frame that is more efficient and faster than the traditional method of scanning the parameter spectrum. They computed a forced libration amplitude of $\theta=-1.14^{\circ} \pm 0.03^{\circ}$ together with a GCP network of 685 points.

Lainey et al. (2021) developed new ephemerides of Phobos and Deimos that are fitted to data including 14 years of MEX data. During the fitting process, they solved for the libration amplitude as $\theta=-1.09^{\circ} \pm 0.01^{\circ}$. Note that this dynamical approach adopts Phobos' gravity field based on the shape model of Willner et al. (2014) and assumes a homogeneous density. The value of Lainey et al. (2021) is close to that of Burmeister et al. (2018), but both values do not agree within their respective error margins.

\section{Mission requirements for MMX geodetic study}

Although the libration amplitude of Burmeister et al. (2018) is consistent with that calculated using a Phobos shape model assuming a uniform density or homogenous internal structure (Willner et al. 2014), possible heterogeneous interiors include layered structures with homothetic boundary shape to the surface because such structures give the same dynamical flattening $\gamma$ and hence do not change the libration amplitude. In order to determine the required accuracy of libration amplitude $\theta$ and degree- 2 gravity coefficients $\left(C_{20}, C_{22}\right)$, we explore a simple two-layer structure that is complementary with models proposed by Le Maistre et al. (2019) (see below). We assume that water ice or porosity is confined to either layer, and calculate how much the MOI deviate from the uniform density case.

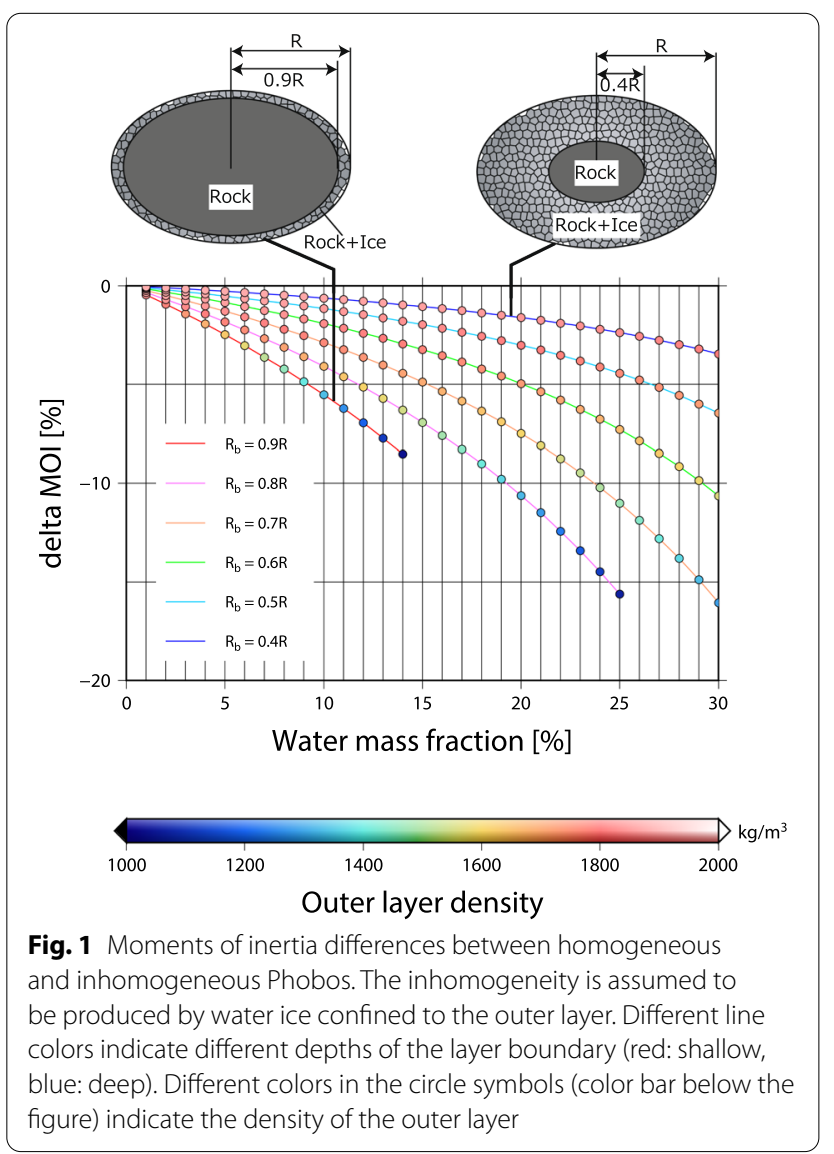




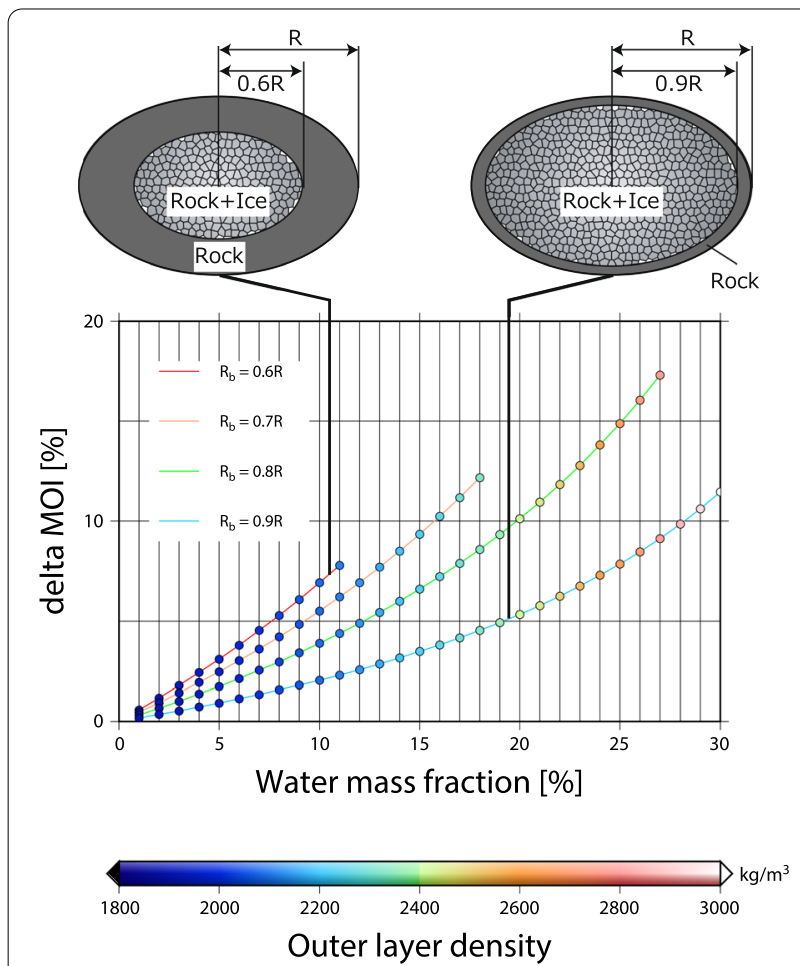

Fig. 2 Same as Fig. 1, but for the case that water ice is confined to the inner layer. Different line colors indicate different depths of the layer boundary (red: deep, blue: shallow)

Phobos' bulk density of $(1847 \pm 12) \mathrm{kg} / \mathrm{m}^{3}$ (section "Bulk density and moments of inertia") is lower than most of the samples of meteoritical carbonaceous material except for the Tagish Lake meteorite, which requires porosity and/or light elements like water ice. If the low bulk density was explained by water ice, its mass fraction is expected to be $10-35 \%$ depending on the rocky material's grain density (Rosenblatt 2011). If the mass distribution inside Phobos is inhomogeneous, e.g., if water ice is concentrated near the surface or the center, we would observe a deviation of the MOI from the value for a homogenous interior. Here, the MOI differences $(\Delta \mathrm{MOI})$ with respect to a homogenous case are calculated assuming a simplified tri-axial ellipsoidal figure of Phobos $a=13.03 \mathrm{~km}, b=11.40 \mathrm{~km}, c=9.14 \mathrm{~km}$ (Willner et al. 2014). Because the percentage of the MOI differences are the same for $x, y, z$ directions under the assumption of similar ellipsoidal boundary shape as the surface, we treat $\Delta \mathrm{MOI}$ as singular value. We assume that water ice is confined either to the outer or to the inner layer and that the rock materials' density is the same for both layers. The water ice mass fraction is varied between 1 and $30 \%$. The libration amplitude for these models is computed as $-1.14739^{\circ}$ and thus consistent with the result of Burmeister et al. (2018). The model-based libration

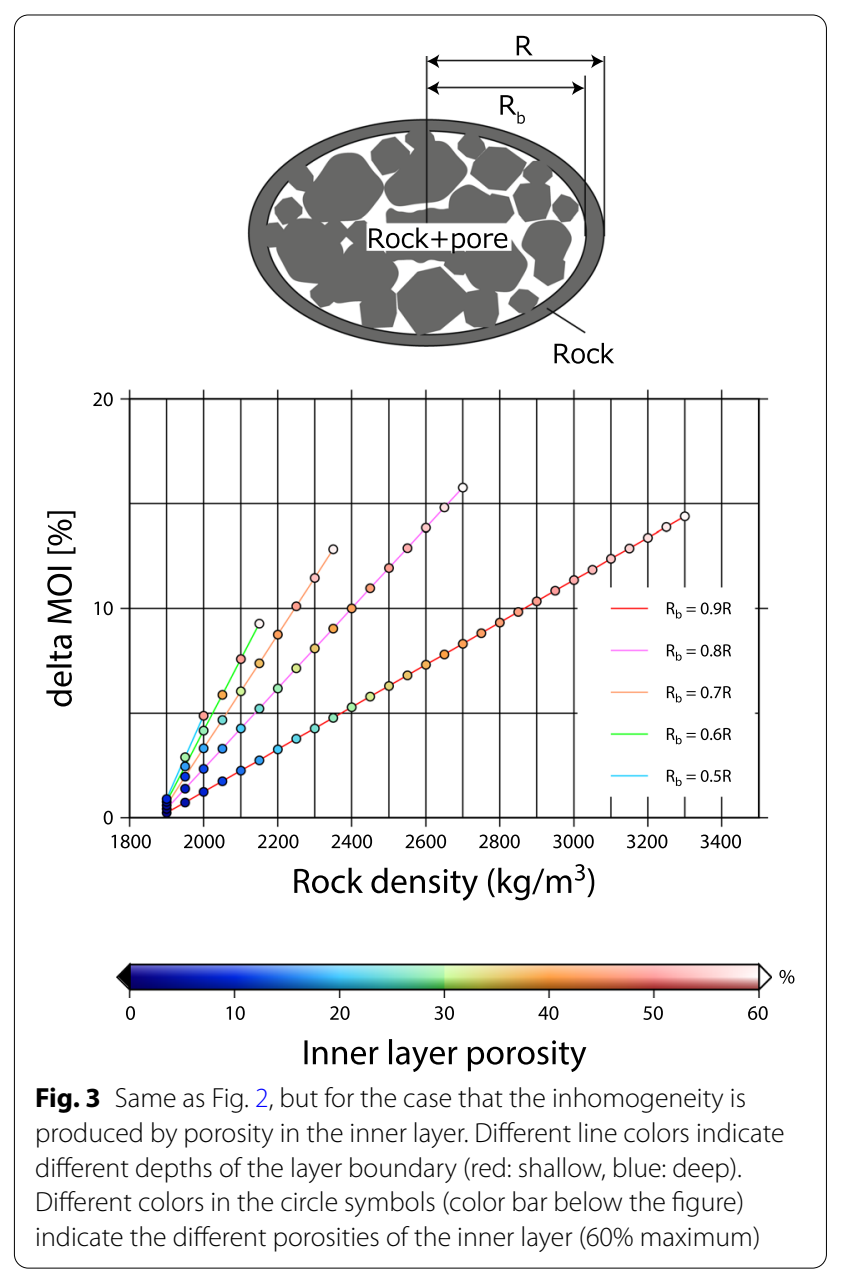

amplitude is computed by discretizing Phobos with 10 -m-size cubes and its uncertainty is $10^{-5}$ degrees that is evaluated as relative error with respect to the value computed with $1 \mathrm{~m}$ cubes.

Figure 1 shows the MOI differences for the case that the outer layer is composed of rock and water ice. When the outer layer thickness is $10 \%$ of the semi-principal axes (labeled as $\mathrm{Rb}=0.9 \mathrm{R}$ ), no more than $14 \mathrm{wt} . \%$ of water ice can be contained in the layer and the maximum $|\Delta \mathrm{MOI}|$ is about $9 \%$. When the layer boundary is deeper, more water ice can be contained, but the maximum $|\Delta \mathrm{MOI}|$ is still about $16 \%$. Figure 2 shows the result for the case in which the water ice is confined to the inner layer. If the layer boundary is located below $0.6 \mathrm{R}$, no more than $11 \mathrm{wt} . \%$ of water ice can be contained in the inner layer and the maximum $|\Delta \mathrm{MOI}|$ is about $8 \%$. When the layer boundary is shallower, more water ice can be contained, but $|\Delta \mathrm{MOI}|$ peaks at about $17 \%$.

We also tested cases in which the porosity is responsible for the low bulk density. We calculated $\Delta$ MOI due to inhomogeneous distribution of the porosity using a 


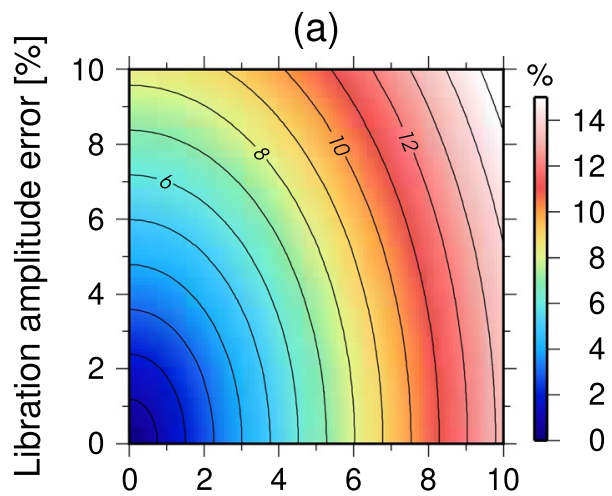

Gravity coefficient error [\%]

(b)

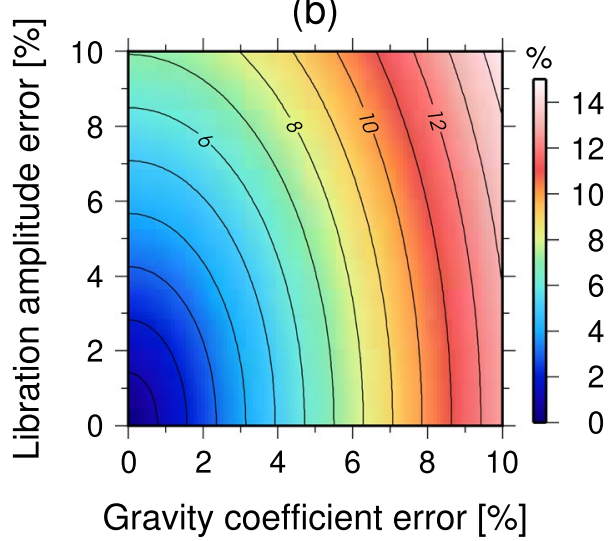

(c)

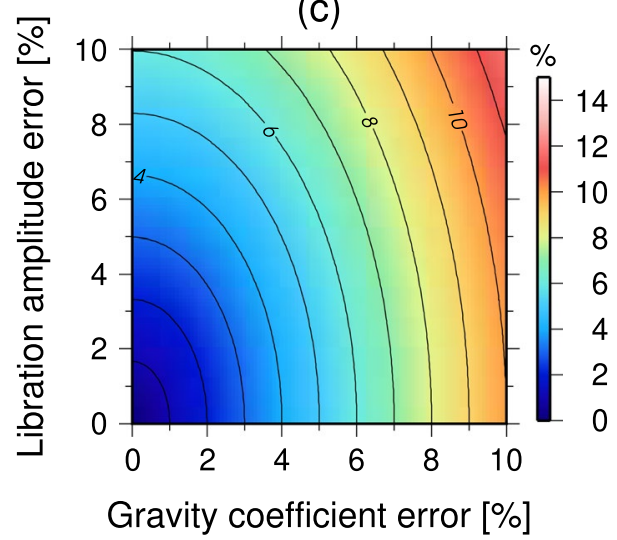

Fig. 4 Error of moments of inertia (MOl) as a function of errors of degree-2 gravity coefficients and libration amplitude. Results for principal moments of inertia $A, B$, and $C$ are shown from top (a) to bottom (c). Color bar indicates the $\mathrm{MOI}$ error in percent

similar two-layer structure. The results depend on the boundary depth and rock density. We assume that the inner layer is porous which is consistent with a formation scenario of Britt et al. (2002) in which the largest pieces reaccreted first after disruption leaving large void inside. If we limit the computed maximum of the porosity as $60 \%$, the maximum $|\Delta \mathrm{MOI}|$ is about $16 \%$ given a boundary position of $0.8 \mathrm{R}$ and a rock density of $2700 \mathrm{~kg} /$ $\mathrm{m}^{3}$ (Fig. 3).

We set the required accuracy of MOI so that we can detect an inhomogeneous density structure due to water ice of $10 \%$ mass fraction that is localized within a relatively small volume of Phobos, i.e., $R_{b}=0.9 \mathrm{R}$ for Fig. 1 and $R_{b}=0.6 \mathrm{R}$ for Fig. 2. Considering $|\Delta \mathrm{MOI}|$ for these situations are $6-8 \%$, the required accuracy for $\mathrm{MOI}$ is $3 \%$. Because the MOI are a function of libration amplitude and gravity coefficients (Eqs. 5-7), errors of MOI are described by propagating the errors of libration amplitude $(\sigma \theta)$ and gravity coefficients $\left(\sigma C_{20}, \sigma C_{22}\right)$ as follows:

$$
\begin{aligned}
& \sigma\left(\frac{A}{M R_{0}^{2}}\right)=\sqrt{\left(\sigma C_{20}\right)^{2}+\left(\frac{2(2-\gamma) \sigma C_{22}}{\gamma}\right)^{2}+\left(\frac{24 e C_{22} \sigma \theta}{\gamma^{2}(3 \theta-6 e)^{2}}\right)^{2},} \\
& \sigma\left(\frac{B}{M R_{0}^{2}}\right)=\sqrt{\left(\sigma C_{20}\right)^{2}+\left(\frac{2(2+\gamma) \sigma C_{22}}{\gamma}\right)^{2}+\left(\frac{24 e C_{22} \sigma \theta}{\gamma^{2}(3 \theta-6 e)^{2}}\right)^{2}}, \\
& \sigma\left(\frac{C}{M R_{0}^{2}}\right)=\sqrt{\left(\frac{4 \sigma C_{22}}{\gamma}\right)^{2}+\left(\frac{24 e C_{22} \sigma \theta}{\gamma^{2}(3 \theta-6 e)^{2}}\right)^{2}} .
\end{aligned}
$$

Figure 4a-c shows the errors of MOI for $A, B$, and $C$ normalized by $M R_{0}^{2}$, respectively. When creating these figures, we assume that $\sigma C_{20}$ and $\sigma C_{22}$ are the same and we used values of $C_{20}, C_{22}$, and $\gamma$ that are based on the homogenous model of Willner et al. (2014). From Fig. $4 a-c$, we can see that it is required to achieve a similar level of accuracy for both the libration amplitude and the gravity coefficients in order to realize a certain accuracy of MOI, otherwise the MOI error is dominated by either component that has the larger error. The required accuracy of the libration amplitude and the degree- 2 gravity coefficient is $2 \%$ for A and B, and $3 \%$ for $\mathrm{C}$.

After we defined the above-mentioned mission requirement using the simplified models in 2015, Le Maistre et al. (2019) presented more detailed and more realistic simulations using 6 different models of possible heterogeneous mass distributions inside Phobos. They computed geodetic observables based on different heterogeneity models by using discretized Phobos models with 500-m-length cubes that fit shape, volume, and total mass, and compared them with the observables based on homogenous mass distribution. Although our two-layer models are simple, they are complementary with the models proposed by Le Maistre et al. (2019) who did not explicitly investigate these two-layer models.

Le Maistre et al. (2019) show that the levels of departure of the MOI due to an interior heterogeneity are smaller than $15 \%$ of their respective homogeneous 
values, which is consistent with our results. Their evenly distributed buried ice (IED) models and disrupted and reaccreted (DAR-F1) models are comparable to our models shown in Fig. 2 (roch + ice) and Fig. 3 (rock+ pore), respectively. The most striking departures from the homogenous case are found for models with mass excess (less porosity) or deficit (more porosity) beneath Stickney crater. They indicate that libration amplitude and low degree gravity coefficients at a precision better than 5\% can allow to identify such heterogeneities, which can be detected if the MMX geodetic mission requirement is met.

\section{Orbits and instruments for MMX geodetic study Quasi-satellite orbit}

The orbit of the spacecraft is fundamental information when observing a planetary body. The spatial resolution of the observation depends on the distance between the spacecraft and the target body. Observation plans are also made based on predicted orbit information. From the viewpoint of global observation, a polar orbit around Phobos is ideal. However, it is impossible for a spacecraft to naturally orbit around Phobos with a Keplerian motion, because Hill's sphere of influence (SOI), that is the region around the body where the dynamics is dominated by its own gravity field, is small for Phobos (Zamaro and Biggs 2015). Therefore, a more stable quasisatellite orbit (QSO) is proposed as the nominal orbit for

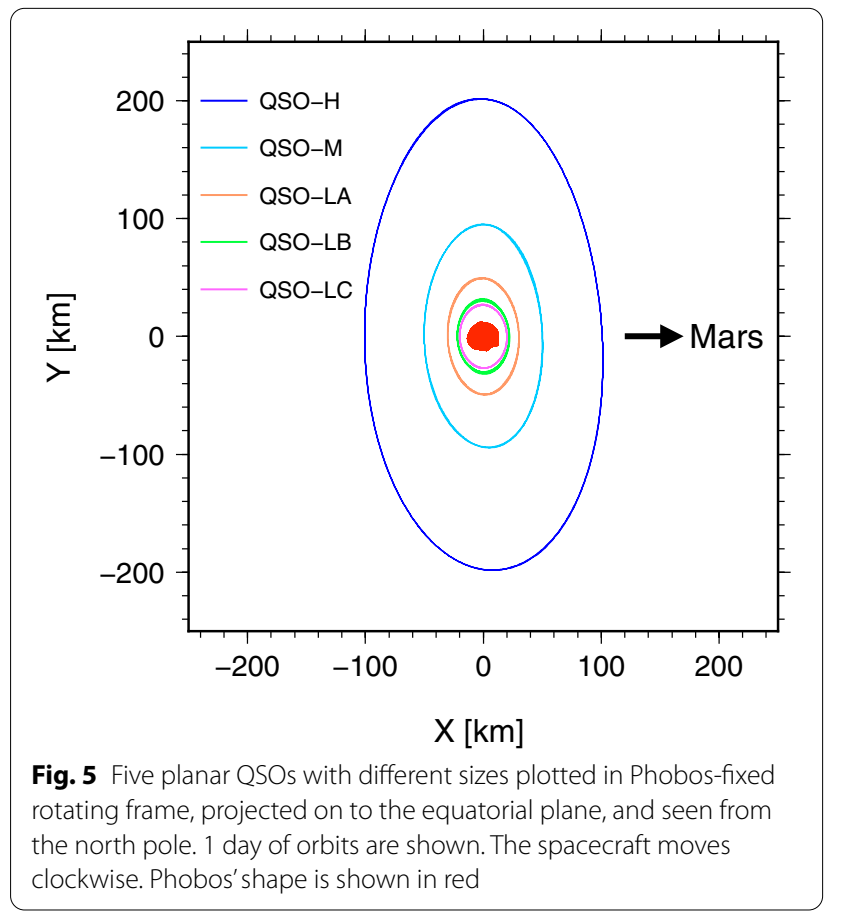

MMX (Ikeda et al. 2017; Baresi et al. 2021). A QSO is a periodic orbit in the circular restricted three-body problem (CR3BP) and is a kind of distant retrograde orbit (DRO) (Lo and Paker 2004). In such a QSO, a spacecraft apparently goes around Phobos in a Phobos-fixed rotating frame, while it actually orbits around Mars with slightly different orbital elements from those of Phobos. A planar orbit is referred to as 2D-QSO (or just QSO for brevity), while one with non-zero out-of-plane component is referred to as $3 \mathrm{D}-\mathrm{QSO}$.

Figure 5 shows proposed planar QSOs with different sizes denoted by pericenter and apocenter distances. The QSOs are roughly divided into three categories, i.e., high (QSO-H, $100 \times 200 \mathrm{~km})$, mid (QSO-M, $50 \times 100 \mathrm{~km})$, and low (QSO-Ls $<50 \mathrm{~km}$ ). The QSO-Ls are further divided into three sub-categories depending on their size (QSO$\mathrm{LA}, \mathrm{LB}$, and $\mathrm{LC}$ ). The planned QSO-H and $-\mathrm{M}$ orbits are nearly ellipses centered on Phobos with an axis ratio of about 1:2. The central distance of the spacecraft is minimum at sub-Mars and anti-sub-Mars points, and maximum at leading and trailing points. For operational safety reasons, the spacecraft will be inserted in a QSO-H first, then gradually lower its altitude. For the QSO-H configuration, the apparent orbital period in the Martian mooncentered Sun-Phobos synodic frame is about 35 days and we will be able to observe the dayside of Phobos for about 2 weeks. In order to provide a shape model during the early mission phase, the current plan is to conduct the main observation for shape modeling at the first QSO-H opportunity (see also Nakamura et al. 2021). Because the planned duration of the first QSO-H is 1 month, we

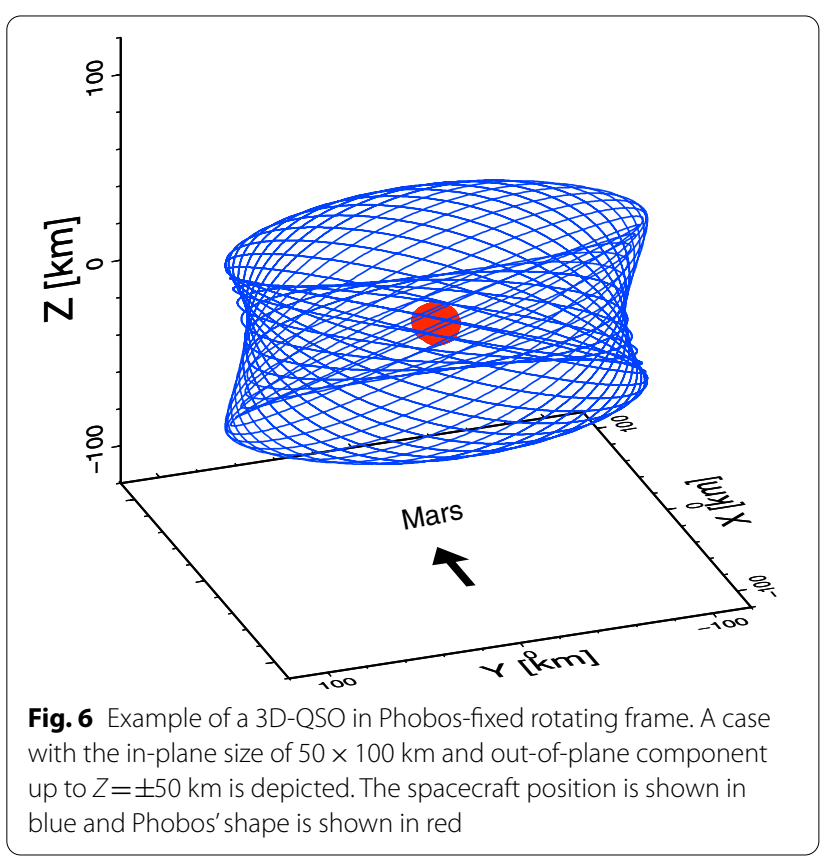


investigate whether necessary observations can be completed within the 2-week dayside period described in "Shape modeling strategy at QSO-H" section.

Because the motion of a spacecraft in a $2 \mathrm{D}-\mathrm{QSO}$ is almost confined to Phobos' equatorial plane, only limited observations for high-latitude and polar regions can be made from $2 \mathrm{D}-\mathrm{QSO}$. However, this situation is greatly mitigated by adopting a $3 \mathrm{D}-\mathrm{QSO}$, thanks to its out-of-plane component. Currently, a 3D-QSO with the equivalent altitude of QSO-M is planned. Figure 6 shows an example of 3D-QSO with about $50-\mathrm{km}$ out-of-plane excursion. The potential observation of polar regions from this 3D-QSO is discussed in "High-latitude and polar observations from 3D-QSO" section.

\section{Mission instruments for geodetic study}

Three mission instruments and one bus instrument will contribute to generating geodetic products. These are the narrow-angle camera TENGOO, the wide-angle camera OROCHI (Kameda et al. 2021), the laser altimeter LIDAR (Senshu et al. 2021), and the narrow-angle camera CAM-T that is mainly used for spacecraft navigation purposes and has similar specifications as TENGOO. TENGOO has a field of view (FOV) of $0.82 \times 1.09 \mathrm{deg}$ and an instantaneous field of view (iFOV) of $5.8 \mu \mathrm{rad} /$ pixel corresponding to $1.2 \mathrm{~m}$ spatial resolution at an altitude of $200 \mathrm{~km}$. For comparison, HRSC onboard MEX has an iFOV of $9 \mu \mathrm{rad} /$ pixel (Oberst et al. 2008) corresponding to $1.8 \mathrm{~m}$ spatial resolution at an altitude of $200 \mathrm{~km}$. Willner et al. (2014) used images from HRSC to reconstruct a shape model, of which spatial resolution varies between 4 and $80 \mathrm{~m}$ depending on flyby distances and channels. The high-resolution images obtained by TENGOO are to be used to update shape models, also for surface studies, landing site evaluation, etc. On the other hand, OROCHI has a wider field of view of $66 \times 53 \mathrm{deg}$ and an iFOV of about $400 \mu \mathrm{rad} /$ pixel corresponding to $80 \mathrm{~m}$ spatial resolution at an altitude $200 \mathrm{~km}$. If an OROCHI image is simultaneously taken with a TENGOO image, it provides important context information to the TENGOO image, i.e., which part of Phobos surface is imaged by TENGOO. Owing to the large field of view of OROCHI, Phobos can be framed within a single OROCHI image if the spacecraft altitude is larger than about $20 \mathrm{~km}$. Therefore, OROCHI images will contribute to spacecraft navigation and orbit determination, i.e., spacecraft attitude information can be obtained from the limb shape and/or the position of landmarks. Because the field of view of TENGOO is narrow, a combination with CAM-T widens apparent field of view if the two cameras are configured with appropriate alignment offset. Such a combination will contribute to reducing the time duration required to cover a certain area of Phobos.
This possibility is discussed in "Spatial and temporal coverage analysis" section.

LIDAR measures the line-of-sight distance between the MMX spacecraft and Phobos' surface by measuring the round-trip travel time of the laser pulse. Its designed measurement range is from $100 \mathrm{~m}$ to $100 \mathrm{~km}$ assuming a geometric albedo of 0.05. Note that Simonelli et al. (1998) reported a globally averaged albedo value of $0.071 \pm 0.012$. The divergence angle of the transmitted beam is 0.5 mrad, which corresponds to a footprint size of $50 \mathrm{~m}$ at an altitude of $100 \mathrm{~km}$. The distance information provided by LIDAR will contribute to improving ephemeris, gravity field, rotational state, and shape of Phobos, as well as the spacecraft orbit. LIDAR range data provide a scale to image-based shape models and cumulative along-track LIDAR footprint (or laser bounce point) positions themselves will provide us high-fidelity geometric height information of Phobos topography. The accuracy of LIDAR measurement almost linearly varies with altitude from \pm $2 \mathrm{~m}$ at an altitude of $100 \mathrm{~m}$ to $\pm 22 \mathrm{~m}$ at an altitude of $100 \mathrm{~km}$ (Senshu et al. 2021). Because the dominant part of the altitude-dependent error is a bias component, the relative along-track topographic profiles can be obtained with an accuracy better than $22 \mathrm{~m}$ even at an altitude of $100 \mathrm{~km}$. The LIDAR measurements will contribute to verifying shape models in terms of topographic features whose horizontal scale is larger than the size of the LIDAR footprint. In addition to data from the onboard instruments, we will also use ground-based radio-tracking data (range and range rate) for geodetic study.

Although it is not directly related to geodetic observation, the mass spectrum analyzer (MSA) (Yokota et al. 2021) may contribute to the interpretation of the geodetic observations in terms of internal structure. Fanale and Salvail $(1989,1990)$ theoretically predicted that the current $\mathrm{H}_{2} \mathrm{O}$ loss rate can be up to $3 \mathrm{~g} / \mathrm{s}\left(\sim 10^{23}\right.$ molecules/s) depending on porosity and pore size. Such sublimation will generate $\mathrm{a}_{2} \mathrm{O}$ gas torus along Phobos' orbit (Mura et al. 2002). MSA can detect such water molecules coming from Phobos' interior if the loss rate is larger than $10^{22}$ molecules/s. Fanale and Salvail $(1889,1990)$ also calculated the latitude-dependent water ice depth that also depends on porosity and pore size. If MSA detected water molecules from Phobos' interior, this information would help us construct interior models of the water ice distribution based on the MSA observation, geodetic observations, and the theoretical studies.

\section{Strategies for observations and data analysis Shape modeling strategy at QSO-H}

Because landing on Phobos is scheduled around the middle of about 3-year staying period around Mars (Nakamura et al. 2021), shape models are to be generated from 


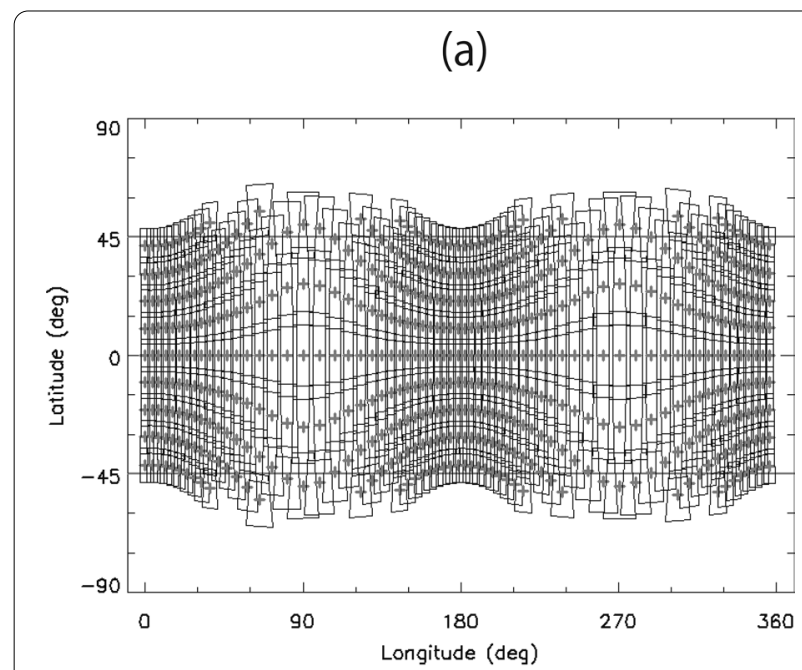

(b)

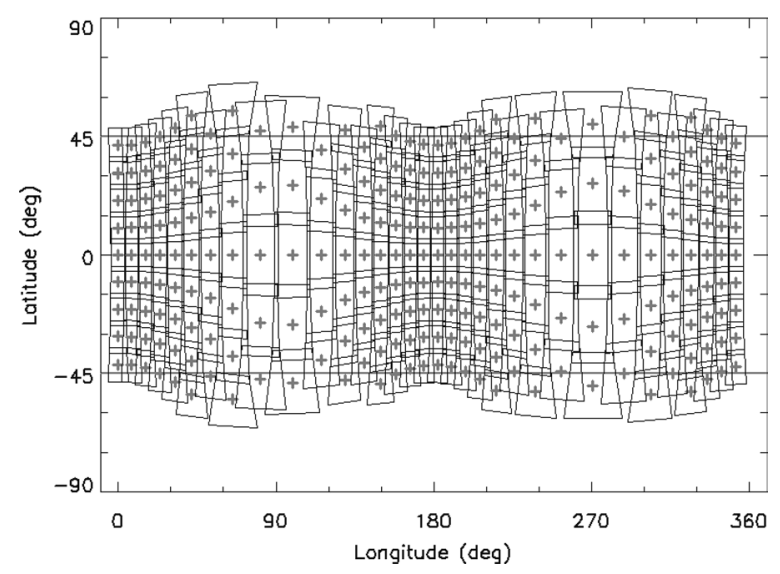

Fig. 7 Simulated field of view of the telescopic camera TENGOO projected on Phobos' surface. Top (a) coverage achieved by TENGOO and bottom (b) coverage achieved by an imaginary camera that mimics a combination of TENGOO and CAM-T with effective longitudinal field of view almost doubled. Observations from equatorial QSO-H with an off-nadir pointing to cover a $\pm 45^{\circ}$ latitudinal band are planned

images collected by the MMX spacecraft from a QSO during early mission phases. This section describes the currently planned strategy for MMX shape modeling by images from QSO-H. In contrast to the current situation of having a limited number of Phobos images with inhomogeneous spatial resolution (Karachevtseva et al. 2014; Witasse et al. 2014), MMX will provide a larger number of images with more homogenous quality and resolution. An existing shape model will be used as initial base model to which our first update will be made with telescopic camera images to be acquired during the terminal

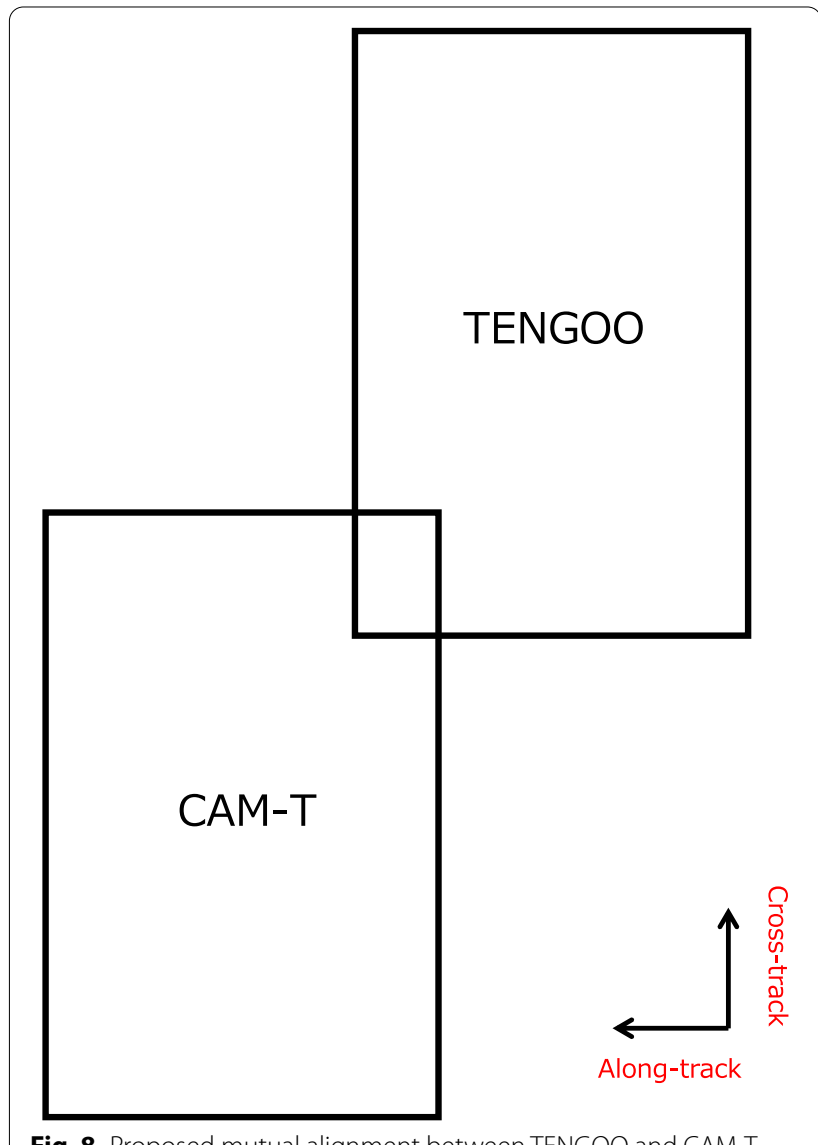

Fig. 8 Proposed mutual alignment between TENGOO and CAM-T

rendezvous phase from a few thousand kilometers distance, with entire or part of Phobos being framed within the field of view of the camera. Due to Phobos' synchronous rotation, only certain hemisphere can be imaged, but these new data would nevertheless contribute to update the initial model. The following two subsections describe preliminary coverage analyses for topography reconstruction from images taken from QSO-H.

\section{Spatial coverage analysis}

Because spacecraft nadir nominally directs to the equator during 2D-QSO periods, we need to slew the spacecraft attitude in latitudinal direction to observe non-equatorial regions. We will first focus on covering equator to midlatitude region. Note that possible landing site candidates are to be selected within $\pm 30^{\circ}$ latitudinal band. Figure $7 \mathrm{a}$ shows an example of multiple TENGOO field of view to cover a $\pm 45^{\circ}$ latitudinal band which is calculated based on a simulated QSO-H trajectory, the TENGOO field of view parameters, and planetographic latitude calculated with a flattening of 0.3. Note that we will make the long side of the rectangle field of view aligned with cross-track (or latitudinal) direction in 2D-QSO operations. The $\pm 45^{\circ}$ 
latitudinal band is covered by 5 to 9 TENGOO images for a certain longitudinal section depending on the longitude (or spacecraft altitude). In total, 508 images from 66 longitudinal sections are necessary to cover the latitudinal band with a $15 \%$ of FOV overlap between adjacent images. When similar observations are made from QSO$\mathrm{M}$, these numbers increase to 8-20 TENGOO images per longitudinal section and 2459 images from 154 sections (not shown).

As will be shown in "Spatial and temporal coverage analysis" section, the first dayside observation opportunity from QSO-H will be too short to cover enough images for shape modeling. One idea to overcome this situation is to spatially complement the insufficiency by making use of CAM-T together with TENGOO. If the fields of view of the two cameras are configured as indicated in Fig. 8, it will result in almost doubled viewing width for both the along-track (longitudinal) and cross-track (latitudinal) directions when scanned in cross-track and along-track directions, respectively. For the current case of taking images of non-equatorial regions from $2 \mathrm{D}-\mathrm{QSO}$ by slewing the spacecraft attitude in cross-track direction, the camera alignment offset in the along-track direction takes effect in extending longitudinal field of view. Figure $7 \mathrm{~b}$ shows the result of a simplified coverage analysis where the viewing width is set to $1.52^{\circ}$ instead of $0.82^{\circ}$ assuming a FOV overlap of $15 \%$. The number of longitudinal sections needed is reduced from 66 to 33 and the total number of images needed to cover the regions of interest is reduced from 508 to 262. In fact, the total number of images does not change so much because TENGOO and CAM-T individually provide images, but the wider area covered by a single favorable observation opportunity will make it possible to complete the imaging operations required for shape modeling within the first dayside period of QSO-H (see next subsection).

\section{Spatial and temporal coverage analysis}

This subsection focuses on whether observations from QSO-H that are necessary for shape modeling are completed within the first dayside period of about 2 weeks. The shape model construction technique of SPC is one of the candidates to be applied to MMX. This technique has been used for numerous celestial bodies, e.g., 25143 Itokawa (Gaskell et al. 2008), 162173 Ryugu (Watanabe et al. 2019), 1 Ceres (Park et al. 2019), Phobos and Deimos, (Ernst et al., submitted to Earth Planets and Space)

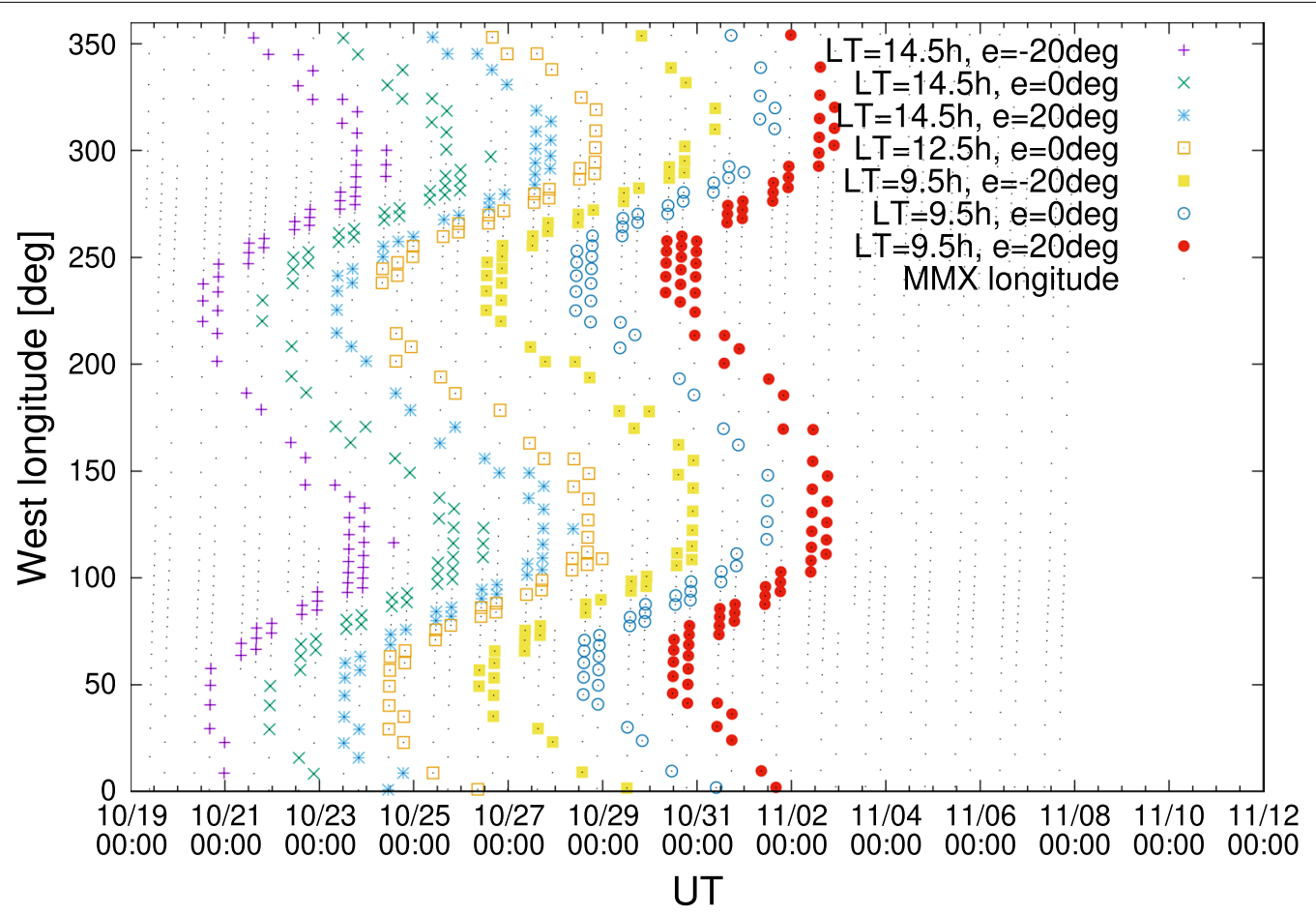

Fig. 9 Favorable observation timings for 7 different conditions. Pluses: looking at local time (LT) $14.5 \mathrm{~h}$ from east, crosses: looking at LT=14.5 h from the meridian, asterisks: looking at $L T=14.5 \mathrm{~h}$ from west, open boxes: looking at $L T=12.5 \mathrm{~h}$ from the meridian, filled boxes: looking at $L T=9.5 \mathrm{~h}$ from east, open circles: $L T=9.5 \mathrm{~h}$ from the meridian, filled circles: looking at $L T=9.5 \mathrm{~h}$ from west. The viewing angle (emission angle) from east and west is set to $20^{\circ}$ at the equator. Small dots indicate the nadir longitude of MMX spacecraft every $10 \mathrm{~min}$. Data during the bus operation period are excluded from the plot 
etc. Here we assume that this technique is applied for MMX shape modeling. Because SPC requires variations in both emission (viewing) and incidence (illumination) angles, we plan to observe a certain area on Phobos surface at least seven times with the following conditions: (1) three times from east, west, and the meridian of the target area at local time around $9.5 \mathrm{~h}$; (2) three times from east, west and the meridian directions at local time around $14.5 \mathrm{~h}$, and (3) once from the meridian around noon (12.5 h).

We search for timings of favorable observations that meet the above conditions within certain tolerance, i.e., we focus on a surface point on the equator at the target's local time and calculate the emission angle at a certain epoch. On the other hand, the whole Phobos is split into 60 sectors each covering a longitude interval of $6^{\circ}$ that corresponds to the longitude width covered by TENGOO at the lowest altitude of QSO-H. The temporal resolution of the analysis is set to $10 \mathrm{~min}$, considering the time required for the cross-track attitude slewing plus margin. The spacecraft position at a certain epoch

(a)

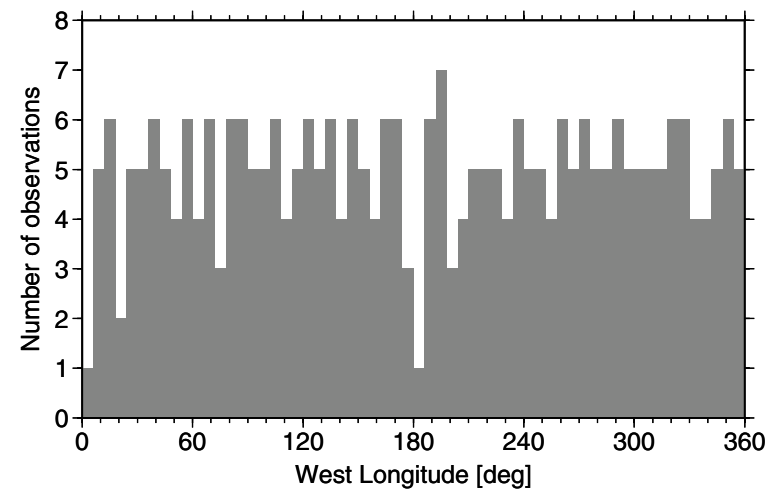

(b)

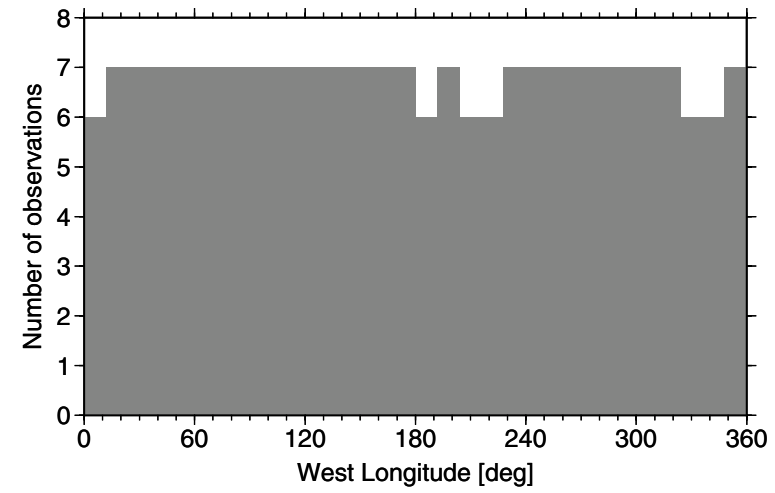

Fig. 10 Number of observation conditions (up to 7) realized for each longitudinal sector: a TENGOO only case and b TENGOO plus CAM-T case. The latter case assumes that the longitudinal FOV is doubled is judged as "favorable" when the emission angle is $20 \pm 5$ degrees at the equator. With this condition met, the longitudinal sector, on which the equatorial point at target local time falls, is marked as "observed". The spacecraft cannot observe Phobos all the time because the spacecraft-fixed high-gain antenna is supposed to point to Earth during periods reserved for bus operations including telecommanding, spacecraft tracking and science/ engineering telemetry downlink. The required time is estimated to be $8 \mathrm{~h} /$ day, which is excluded from our analysis as "unobservable" period that is set from 0 to $8 \mathrm{UT}$ considering the visibility condition of the spacecraft from a tracking station in Japan. Plotted in Fig. 9 are the timing for the seven "favorable" observation opportunities and "observed" longitude, which shows that the observation opportunities are temporally well separated over the dayside period. However, there are many longitudinal sectors that are not observed seven times during the first QSO-H dayside period as shown in Fig. 10a. The situation is more severe for longitudes around 0 and $180^{\circ}$ (Mars and anti-Mars sides) where the spacecraft's altitude becomes minimum and the resultant surface area covered by TENGOO's FOV becomes the smallest. The spacecraft velocity relative to Phobos' surface is also maximum at these longitudes. Next, we analyzed the "TENGOO plus CAM-T" case where the width of the longitudinal sector is set to $12^{\circ}$ and the result is shown in Fig. 10b. In this case, almost all of the longitudinal sectors are observed with the seven measurement opportunities, which means that the necessary observations can be mostly completed within the first dayside period by combining TENGOO with CAM-T. We plan to fill the remaining observation gaps in the subsequent QSO-M period (see Fig. 1 of Nakamura et al. 2021).

The above analysis is based on a simplified spheroidal shape of Phobos. The real topography of Phobos shows large deviations from the spheroid, e.g., inside Stickney, a large crater of $9 \mathrm{~km}$ across. A more detailed analysis is necessary for such regions because incidence and emission angles show complex variations due to the topographic slopes.

Images with higher spatial resolution are to be obtained from QSOs with lower altitude than QSO-H. The survey for landing site candidates is planned from QSO-M, and more detailed observations will be made from QSO-Ls for further down-selected candidates. Local digital terrain models will be developed by SPC and/or stereo photogrammetry using TENGOO/CAM-T images from these low-altitude QSOs.

\section{High-latitude and polar observations from 3D-QSO}

A 3D-QSO provides us with precious opportunities to observe high-latitude and polar regions of Phobos. The 


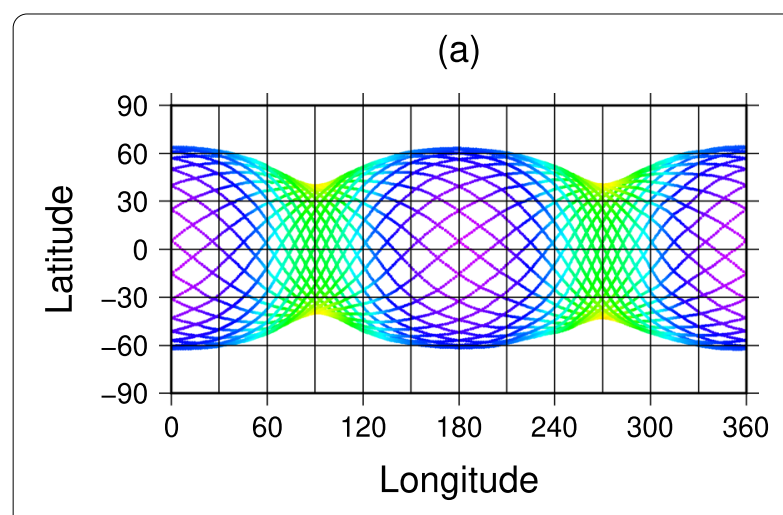

(b)
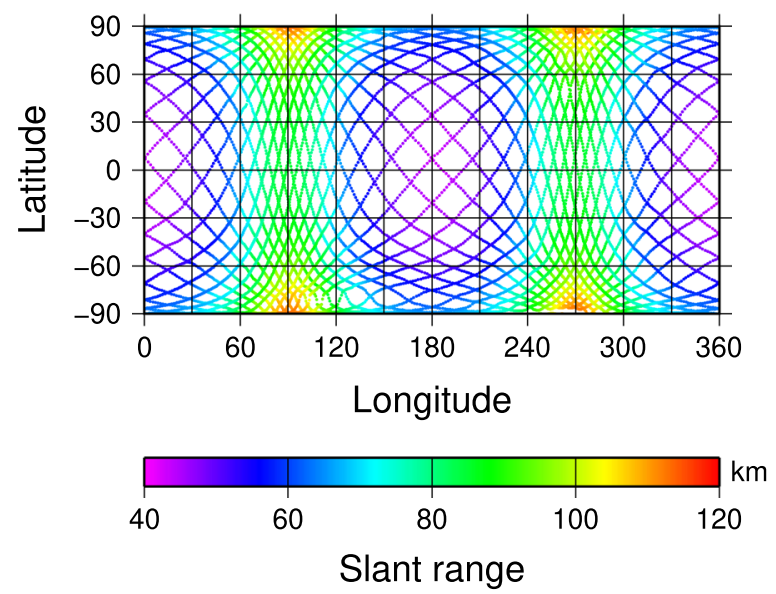

Fig. 11 Top (a) nadir-pointing spacecraft ground tracks for the 3D-QSO shown in Fig. 6. Bottom (b) ground tracks making polar observations possible by formulating the slewing angle as a function of the Z-component of spacecraft position. The orbits are plotted for a span of 10 days

observability depends on the amount of the out-of-plane excursion that correlates with delta- $\mathrm{V}$ amount required to transfer from 2D-QSO. Currently an excursion from planar QSO-M up to about $50 \mathrm{~km}$ is proposed (Fig. 6). Figure 11a shows the nadir-pointing ground tracks with the latitude being planetographic (flattening $=0.3$ ). For this particular 3D-QSO, the ground track repeat cycle is about 9 days. On the other hand, the nadir illumination conditions vary with a period of about 1 week and it is found that, if the observation is restricted to the dayside region only, the corresponding apparent repeat cycle is about 1 month.

Figure 11a indicates that an off-nadir pointing is necessary to look at the poles. The required slewing angle depends on the spacecraft position. Figure 11b shows an example of a ground track that makes polar observations possible by formulating the slewing angle as a function of Z-component of spacecraft position (or the out-of-plane component). The illumination condition of the polar regions varies with a beta angle that has seasonal variations with an amplitude of about $25^{\circ}$, where the beta angle is the angle between the orbital plane of Phobos and the vector to the Sun or the latitude of the sub-solar point on Phobos. The MMX spacecraft will be inserted into QSO-LB or LC when the south polar region is illuminated with a beta angle about $-25^{\circ}$ around May 2026 and landing operations is planned around April 2027 when the north polar region is illuminated with a beta angle of $25^{\circ}$. The envisioned time frame of the 3D-QSO is around October 2026 when the beta angle is close to zero (Nakamura et al. 2021). The beta angle would change by about $6^{\circ}$ if the timeline shifted by a month. Such a low absolute value of the beta angle is suitable to get a first overview of both poles. However, the incidence angle becomes large in the polar regions, which makes topography reconstruction from images difficult. Although its spatial resolution is lower than that of images, LIDAR data will help reconstruct the topography of polar regions. The maximum slant range is about $120 \mathrm{~km}$ that exceeds the designed LIDAR range capability of $100 \mathrm{~km}$, but the designed capability is based on a geometric albedo of 0.05 that is smaller than the global average of 0.07 . If the polar albedo is equal to or larger than the global average, the distance attenuation of reflected laser power can be compensated for, making LIDAR observation possible, albeit not perfect due to yet large incidence angle of the LIDAR measurements.

\section{Strategies for ephemeris, gravity field, and rotation parameter of Phobos}

Because the orbit plane of the MMX spacecraft is close to that of Phobos having a small inclination with respect to Mars equatorial plane, the spacecraft velocity change would not be perpendicular to the line-of-sight direction to the Earth. It is expected that spacecraft orbit around Mars will be well determined from Earth-based range and Doppler observations with good sensitivity owing to the above geometry. The Phobos ephemeris can be improved by combining the spacecraft position in the inertial frame determined by the range and Doppler data with targetrelative LIDAR and image data. Most significant error in the mean motion (Jacobson and Laney 2014) is expected to be reduced. Phobos ephemeris improvement will be enabled by operating LIDAR from QSO-M and -L with even better performances if radio-tracking of the spacecraft (range and Doppler measurements) using mediumgain antenna is carried out simultaneously with LIDAR ranging. Yang et al. (2019) estimated errors of degree-2 gravity coefficients introduced by error in Phobos ephemeris, which was no larger than $0.52 \%$ based on an 
assumed shift of $200 \mathrm{~m}$. If true ephemeris error was less than about $1 \mathrm{~km}$, the corresponding gravity coefficients error would be smaller than the required accuracy.

Because the gravity field perturbs the spacecraft orbit, a precise orbit determination improves the estimation of the gravity coefficients. From the viewpoint of gravity field recovery, a smaller size of QSO or lower spacecraft altitude is preferable because a larger sensitivity to the gravity field is gained by shorter distance between the spacecraft and the target. In this regard, QSO-H provides only weak sensitivities to gravity coefficients because the size of QSO-H is larger than 9 times of Phobos mean radius $(\sim 11 \mathrm{~km})$. Spacecraft tracking at QSO-M will allow us to better retrieve $C_{22}$, and $3 \mathrm{D}-\mathrm{QSO}$ at this altitude will contribute to solving for zonal $C_{20}$ with smaller correlation with $C_{22}$. Further observation at QSO-Ls, in particular the planned lowest one QSO-LC of $20 \times 27 \mathrm{~km}$ in size with a minimum altitude of about $7 \mathrm{~km}$, provides a better opportunity for estimating $C_{22}$ and possibly other low-degree sectorial coefficients. The best situation for the gravity field recovery would be given by a 3D-QSO at such a low altitude, but the feasibility depends on the stability of the orbit and the safety of the spacecraft.

In addition to the conventional Earth-based 2-way Doppler and range observation, LIDAR data and optical landmark tracking data are to be incorporated into the orbit determination process to improve its accuracy. The LIDAR observation provides target-relative line-of-sight distance to Phobos, and the optical observation provides pixel and line location of a landmark in the image which has sensitivity in the direction perpendicular to the line-of-sight direction. The two observations play complementary roles in the orbit determination process (Yamamoto et al. 2020).

The acceleration on the spacecraft caused by the gravity coefficients is calculated in the body-fixed coordinate system. If the axes of the body-fixed frame are defined by the principal axes of the body, $C_{21}, S_{21}$ and $S_{22}$ disappear. A precise shape model is needed to translate the targetrelative LIDAR measurements to the Phobos center of mass $(\mathrm{COM})$ that is necessary for the spacecraft orbit determination and Phobos ephemeris determination. The offset between center of mass and center of figure (COF) will be first calculated from the image-based shape model by assuming constant density, based on which the orbit and gravity analysis will be conducted. The resultant degree one gravity coefficient will be used to redefine the COM-COF offset for subsequent iterative analyses.

The main error sources for a SPC shape model are the uncertainties in the camera position (or spacecraft position) and camera pointing direction, which are difficult to separate because the image data alone are not sensitive to radial position of the camera (Park et al. 2019). Errors in rotation parameters (e.g., libration amplitude) will also degrade the shape modeling accuracy. If the orbit determination process can estimate the spacecraft state, gravity field, rotation parameters, and camera pointing directions, the shape modeling results will be improved by these information, which will in turn update the orbit determination results by iterative procedures. Such geodetically derived information will contribute to selecting potential landing sites as well as to the planning of the landing operation itself.

\section{Summary}

This paper described the accuracy of libration amplitude and degree- 2 gravity coefficients that is necessary to meet a part of MMX mission requirements. We studied how the MOI changes due to an inhomogeneous distribution of water ice or porosity with respect to the MOI for a homogenous interior of Phobos. The calculation is based on a simple two-layer model having the similar boundary figure to the surface, which is consistent with the currently observed libration amplitude. In such a configuration, the maximum deviation of the MOI is smaller than $17 \%$. In order to detect the presence of inhomogeneity in the density structure due to localization of water ice of $10 \%$ of Phobos' mass, it is required to achieve at least $3 \%$ of MOI accuracy. To this end, the required accuracy for the libration amplitude and the degree- 2 gravity coefficients are $2-3 \%$.

The data from three cameras of TENGOO, OROCHI, CAM-T and laser altimeter LIDAR, together with Earth-based radio-tracking data, will contribute to improving our knowledge of ephemeris, gravity field, shape, and rotation parameter of Phobos. We presented the preliminary imaging strategy for creating a base shape model of the equatorial to mid-latitude region of Phobos that is required for landing site selection process at the early stage of the mission. The accuracies of the geodetic products are expected to be improved by an iterative process of orbit determination and shape modeling. Such products will contribute to achieving the MMX mission goals by providing basic and important information for interpreting data from MMX mission instruments and for selecting the landing site.

\section{Acknowledgements \\ We thank Naoki Terada for discussions on MSA observation. We thank three anonymous reviewers for their thoughtful and constructive comments.}

Authors' contributions

Design of the work: KM. Manuscript preparation: KM and NH. QSO design: HK and NB. Observation strategy: KM, TK, NH, HS. All authors read and approved the final manuscript.

Funding

Not applicable. 


\section{Availability of data and materials}

The datasets used and/or analyzed during the current study are available from the corresponding author on reasonable request.

\section{Declarations}

\section{Competing interests}

The authors declare that they have no competing interests.

\section{Author details}

${ }^{1}$ RISE Project, National Astronomical Observatory of Japan, Oshu, Iwate 023-0861, Japan. ${ }^{2}$ The Graduate University for Advanced Studies, SOKENDAl, Hayama, Kanagawa 240-0193, Japan. ${ }^{3}$ University of Aizu, Aizu-Wakamatsu 965-8580, Japan. ${ }^{4}$ Institute of Space and Astronautical Science, Japan Aerospace Exploration Agency, Sagamihara, Kanagawa 252-0222, Japan. ${ }^{5}$ National Institute of Advanced Industrial Science and Technology, Tsukuba 305-8560, Japan. ${ }^{6}$ Planetary Exploration Research Center, Chiba Institute of Technology, Tsudanuma, Narashino, Chiba 275-0016, Japan. ${ }^{7}$ RISE Project, National Astronomical Observatory of Japan, Mitaka, Tokyo 181-8588, Japan. ${ }^{8}$ University of Tokyo, Tokyo 113-0033, Japan. ${ }^{9}$ Earthquake Research Institute, University of Tokyo, Tokyo 113-0032, Japan. ${ }^{10}$ Hokkaido University, Sapporo 060-0810, Japan. ${ }^{11}$ Present Address: University of Surrey, Guildford GU2 $7 X \mathrm{XH}$, Surrey, UK.

\section{Received: 11 January 2021 Accepted: 16 August 2021}

Published online: 15 December 2021

\section{References}

Andert TP, Rosenblatt P, Pätzold M, Häusler B, Dehant V, Tyler GL, Marty JC (2010) Precise mass determination and the nature of Phobos. Geophys Res Lett 37:L09202. https://doi.org/10.1029/2009GL041829

Ballouz RL, Baresi N, Crites ST, Kawakatsu Y, Fujimoto M (2019) Surface refreshing of Martian moon Phobos by orbital eccentricity-driven grain motion. Nat Geosci 12:229-234. https://doi.org/10.1038/ s41561-019-0323-9

Baresi N, Dei Tos DA, Ikeda H, Kawakatsu Y (2021) Trajectory design and maintenance of the Martian moons eXploration mission around Phobos. J Guid Control Dyn. https://doi.org/10.2514/1.G005041

Bills BG, Rubincam DP (1995) Constraints on density models from radial moments: applications to Earth, Moon, and Mars. J Geophys Res 100(E12):26305-26315. https://doi.org/10.1029/95JE02776

Borderies N, Yoder CF (1990) Phobos' gravity field and its influence on its orbit and physical librations, Astron. Astrophys 233:235-251

Britt DT, Yeomans D, Housen K, Consolmagno G (2002) Asteroid density, porosity and structure. Asteroids III:485-500

Burmeister S, Willner K, Schmidt V, Oberst J (2018) Determination of Phobos' rotational parameters by an inertial frame bundle block adjustment. J Geod 92:963-973. https://doi.org/10.1007/s00190-018-1112-8

Craddock RA (2011) Are Phobos and Deimos the result of a giant impact? Icarus 211:1150-1161. https://doi.org/10.1016/j.icarus.2010.10.023

Duxbury TC, Callahan JD (1989) Phobos and Deimos control networks. Icarus 77:275-286

Ernst CM, Barnouin OS, Daly RT, SBMT team (2018) The Small Body Mapping Tool (SBMT) for accessing, visualizing, and analyzing spacecraft data in three dimensions, Lunar and Planetary Science Conference 1043

Fanale FP, Salvail JR (1989) Loss of water from Phobos. Geophys Res Lett 16:287-290. https://doi.org/10.1029/GL016i004p00287

Fanale FP, Salvail JR (1990) Evolution of the water regime of Phobos. Icarus 88:380-395. https://doi.org/10.1016/0019-1035(90)90089-R

Gaskell RW, Barnouin-Jha OS, Scheeres DJ, Konopliv AS, Mukai T, Abe S, Saito J, Ishiguro M, Kubota T, Hashimoto T, Kawaguchi J, Yoshikawa M, Shirakawa K, Kominato T, Hirata N, Demura H (2008) Characterizing and navigating small bodies with imaging data. Meteorit Planet Sci 43:1049-1061

Gaskell RW (2011) Phobos Shape Model V1.0, NASA Planetary Data System VO1-SA-VISANISB-5-PHOBOSSHAPE-V1.0

Hu X, Oberst J, Willner K (2020) Equipotential figure of Phobos suggests its late accretion near 3.3 Mars radii. Geophys Res Lett 47:958. https://doi.org/10. 1029/2019GL085958
Ikeda H, Mitani S, Mimasu Y, Ono G, Nigo K, Kawakatsu Y (2017) Orbital Operations Strategy in the Vicinity of Phobos, Proceedings of the 26th International Symposium on Space Flight Dynamics /31st International Symposium on Space Technology and Science, Matsuyama, ISSFD-2017008 / ISTS-2017-d-008

Jacobson RA (2010) The orbits and masses of the Martian satellites and the libration of Phobos. Astron J 139:668-679. https://doi.org/10.1088/0004$6256 / 139 / 2 / 668$

Jacobson RA, Lainey V (2014) Martian satellite orbits and ephemerides. Planet Space Sci 102:35-44. https://doi.org/10.1016/j.pss.2013.06.003

Kameda S et al (2021) Design of telescopic nadir imager for geomorphology (TENGOO) and observation of surface reflectance by optical chromatic imager (OROCHI) for the Martian Moons Exploration (MMX). Earth Planets Space. https://doi.org/10.1186/s40623-021-01462-9

Karachevtseva IP, Oberst J, Zubarev AE, Nadezhdina IE, Kokhanov AA, Garov AS, Uchaev DV, Uchaev DV, Malinnikov VA, Klimkin ND (2014) The Phobos information system. Planet Space Sci 102:74-85. https://doi.org/10.1016/j. pss.2013.12.015

Kawakatsu Y, Kuramoto K, Ikeda H, Ozaki N, Baresi N, Ono G, Tsui T, Imada T, Shimada T, Kusano H, Sawada H, Ozawa T, Baba M, Otake H (2018) Mission Design of Martian Moons eXploration (MMX), in 69th International Astronautical Congress, IAC-18-A3.3A.8

Konopliv AS, Park RS, Vaughan AT, Bills BG, Asmar SW, Ermakov Al, Rambaux N, Raymond CA, Castillo-Rogez JC, Russell CT, Smith DE, Zuber MT (2018) The Ceres gravity field, spin pole, rotation period and orbit from the Dawn radiometric tracking and optical data. Icarus 299:411-429. https:// doi.org/10.1016/j.icarus.2017.08.005

Kuramoto Ket al (2021) Martian moons exploration MMX: sample return mission to Phobos elucidating formation processes of habitable planets. Earth Planets Space. https://doi.org/10.1186/s40623-021-01545-7

Lainey V, Pasewaldt A, Robert V, Rosenblatt P, Jaumann R, Oberst J, Roatsch T, Willner K, Ziese R, Thuillot W (2021) Mars moon ephemerides after 14 years of Mars Express data. Astron Astrophys. https://doi.org/10.1051/ 0004-6361/202039406

Lo M, Parker J (2004) Unstable resonant orbits near earth and their applications in planetary missions. AIAA/AAS Astrodyn Special Conf Exhib. https://doi.org/10.2514/6.2004-5304

Le Maistre S, Rivoldini A, Rosenblatt P (2019) Signature of Phobos'interior structure in its gravity field and libration. Icarus 321:272-290. https://doi. org/10.1016/j.icarus.2018.11.022

Matsumoto K, Noda H, Ishihara Y, Senshu H, Yamamoto K, Hirata N, Hirata N, Namiki N, Otsubo T, Higuchi A, Watanabe S, Ikeda H, Mizuno T, Yamada R, Araki H, Abe S, Yoshida F, Sasaki S, Oshigami S, Tsuruta S, Asari K, Shizugami M, Yamamoto Y, Ogawa N, Kikuchi S, Saiki T, Tsuda Y, Yoshikawa M, Tanaka S, Terui F, Nakazawa S, Yamaguchi T, Takei Y, Takeuchi H, Okada T, Yamada M, Shimaki Y, Shirai K, Ogawa K, lijima Y (2020) Improving Hayabusa2 trajectory by combining LIDAR data and a shape model. Icarus 338:113574. https://doi.org/10.1016/j.icarus.2019.113574

Miyamoto H, Yano H, Scheeres DJ, Abe S, Barnouin-Jha O, Cheng AF, Demura H, Gaskell RW, Hirata N, Ishiguro M, Michikami T, Nakamura AM, Nakamura R, Saito J, Sasaki S (2007) Regolith migration and sorting on asteroid Itokawa. Science 316(5827):1011-1014. https://doi.org/10. 1126/science.1134390

Mohr PJ, Newell DB, Taylor BN (2016) CODATA recommended values of the fundamental physical constants: 2014. Rev Mod Phys 88:035009. https://doi.org/10.1103/RevModPhys.88.035009

Mura A, Milillo A, Orsini S, Kallio E, Barabash S (2002) Energetic neutral atoms at Mars, 2, Imaging of the solar wind-Phobos interaction. J Geophys Res 107(A10):1278. https://doi.org/10.1029/2001JA000328

Nakamura T et al (2021) Science operation plan of Phobos and Deimos from the MMX spacecraft. Earth Planets Space. https://doi.org/10.1186/ s40623-021-01546-6

Oberst J, Schwarz G, Behnke T, Hoffmann H, Matz K-D, Flohrer J, Hirsch H, Roatsch T, Scholten F, Hauber E, Brinkmann B, Jaumann R, Williams D, Kirk R, Duxbury T, Leu C, Neukum G (2008) The imaging performance of the SRC on Mars Express. Planet Space Sci 56:473-491

Oberst J, Zubarev A, Nadezhdina I, Shishkina L, Rambaux N (2014) The Phobos geodetic control point network and rotation model. Planet Space Sci 102:45-50

Park RS, Vaughan AT, Konopliv AS, Ermakov Al, Mastrodemos N, CastilloRogez JC, Joy SP, Nathues A, Polanskey CA, Rayman MD, Riedel JE, 
Raymond CA, Russell CT, Zuber MT (2019) High-resolution shape model of Ceres from stereophotoclinometry using Dawn Imaging Data. Icarus 319:812-827. https://doi.org/10.1016/j.icarus.2018.10.024

Pätzold M, Andert TP, Tyler GL, Asmar SW, Häusler B, Tellmann S (2014) Phobos mass determination from the very close flyby of Mars Express in 2010. Icarus 229:92-98. https://doi.org/10.1016/j.icarus.2013.10.021

Rosenblatt P (2011) The origin of the Martian moons revisited. Astron Astrophys Rev 19(1):44

Senshu $\mathrm{H}$ et al (2021) Light detection and ranging (LIDAR) laser altimeter for the Martian Moons Exploration (MMX) spacecraft. Earth Planets Space. https://doi.org/10.1186/s40623-021-01537-7

Shi X, Willner K, Oberst J, Ping J, Ye S (2012) Working models for the gravitational field of Phobos. Sci China Phys Mech Astron 55:358-364. https:// doi.org/10.1007/s11433-011-4606-4

Simonelli DP, Wisz M, Switala A, Adinolfi D, Veverka J, Thomas PC, Helfenstein $P$ (1998) Photometric properties of Phobos surface materials from Viking images. Icarus 131:52-77. https://doi.org/10.1006/icar.1997.5800 Watanabe $S$ et al (2019) Hayabusa2 arrives at the carbonaceous asteroid 162173 Ryugu - a spinning top-shaped rubble pile. Science 364:268272. https://doi.org/10.1126/science.aav8032

Willner K, Oberst J, Hussmann H, Giese B, Hoffmann H, Matz K-D, Roatsch T, Duxbury T (2010) Phobos control point network, rotation, and shape. Earth Planet Sci Lett 294:541-546

Willner K, Shi X, Oberst J (2014) Phobos' shape and topography models. Planet Space Sci 102:51-59

Witasse O, Duxbury T, Chicarro A, Altobelli N, Andert T, Aronica A, Barabash S, Bertaux J-L, Bibring J-P, Cardesin-Moinelo A, Cichetti A, Companys V, Dehant V, Denis M, Formisano V, Futaana Y, Giuranna M, Gondet B, Heather D, Hoffmann H, Holmström M, Manaud N, Martin P, Matz K-D, Montmessin F, Morley T, Mueller M, Neukum G, Oberst J, Orosei R, Pätzold M, Picardi G, Pischel R, Plaut JJ, Reberac A, Pardo Voss P, Roatsch T, Rosenblatt P, Remus S, Schmedemann N, Willner K, Zegers T (2014) Mars Express investigations of Phobos and Deimos. Planet Space Sci 102:18-34. https://doi.org/10.1016/j.pss.2013.08.002

Yamamoto K, Otsubo T, Matsumoto K, Noda H, Namiki N, Takeuchi H, lkeda H, Yoshikawa M, Yamamoto Y, Senshu H, Mizuno T, Hirata N, Yamada R, Ishihara Y, Araki H, Abe S, Yoshida F, Higuchi A, Sasaki S, Oshigami S, Tsuruta S, Asari K, Shizugami M, Ogawa N, Ono G, Mimasu Y, Yoshikawa K, Takahashi T, Takei Y, Fujii A, Yamaguchi T, Kikuchi S, Watanabe S-I, Tanaka S, Terui F, Nakazawa S, Saiki T, Tsuda Y (2020) Dynamic precise orbit determination of Hayabusa2 using laser altimeter (LIDAR) and image tracking data sets. Earth Planets and Space. https://doi.org/10. 1186/s40623-020-01213-2

Yang X, Yan JG, Andert T, Ye M, Pätzold M, Hahn M, Jin WT, Li F, Barriot JP (2019) The second-degree gravity coefficients of Phobos from two Mars Express flybys. Mon Not R Astron Soc. https://doi.org/10.1093/mnras/stz2695

Yokota S et al (2021) In situ observations of ions and magnetic field around Phobos: the mass spectrum analyzer (MSA) for the Martian Moons eXploration (MMX) mission. Earth Planets Space. https://doi.org/10.1186/ s40623-021-01452-x

Zamaro M, Biggs JD (2015) Natural motion around the Martian moon Phobos: the dynamical substitutes of the Libration Point Orbits in an elliptic three-body problem with gravity harmonics. Celest Mech Dyn Astr 122:263-302. https://doi.org/10.1007/s10569-015-9619-2

\section{Publisher's Note}

Springer Nature remains neutral with regard to jurisdictional claims in published maps and institutional affiliations.

\section{Submit your manuscript to a SpringerOpen ${ }^{\circ}$ journal and benefit from:}

- Convenient online submission

- Rigorous peer review

- Open access: articles freely available online

- High visibility within the field

- Retaining the copyright to your article

Submit your next manuscript at $\boldsymbol{\nabla}$ springeropen.com 